\title{
Complex open elbow fracture-dislocation with severe proximal ulna bone loss: a case report of massive osteochondral allograft surgical treatment
}

\author{
Chiara Concina ${ }^{1}$, Marina Crucil ${ }^{1}$, Emmanouil Theodorakis ${ }^{2}$, Giorgio Saggin $^{1}$, Silvia Perin ${ }^{1}$, \\ Franco Gherlinzoni ${ }^{1}$ \\ ${ }^{1}$ Department of Orthopedics and Traumatology, Gorizia-Monfalcone Hospital, Monfalcone, Italy \\ ${ }^{2}$ Department of Orthopedics and Traumatology, San Carlo Borromeo Hospital, Milan, Italy
}

\begin{abstract}
We report a case of a 69-year-old right-dominant man who had an open Monteggia-like lesion of the right elbow (Gustilo-Andersen IIIA) with severe proximal ulna bone loss associated with an ipsilateral ulnar shaft fracture due to a motorcycle accident. The patient underwent two-stage surgery. Wound debridement and bridging external fixation were performed at first. Three months later, a frozen massive osteochondral ulnar allograft was implanted and fixed with a locking compression plate. A superficial wound infection appeared 5 weeks after the second surgery. Superficial wound debridement, negative pressure therapy, and antibiotics were administered for 3 months, achieving infection healing. At 3 years post-surgery, the elbow range of motion was satisfactory with a Disabilities of the Arm, Shoulder and Hand (DASH) score of 16.7. Radiographs and computed tomography scans showed good allograft-bone integration without allograft reabsorption or hardware loosening. Although not complication-free, massive ulna osteochondral allograft implantation can be considered a valid option in cases of open Monteggia-like lesions associated with ulnar shaft fracture and severe bone loss in active patients, whenever osteosynthesis or joint replacement is not a proper solution. This type of bone stock restoration allows for future surgery, if needed.
\end{abstract}

Keywords: Elbow; Fracture dislocation; Monteggia’s fracture; Allografts

Elbow fracture-dislocations are complex injuries in which both bone structure and soft tissue are damaged. These complex injuries can lead to functional disability because of persistent pain, residual instability, or stiffness. For these reasons, fracture-dislocations remain a challenge for orthopedic surgeons. Because the elbow is a superficial joint, high-energy trauma usually leads to comminuted fractures with articular disruption, soft tissue damage, and neurovascular injuries. Bone loss can sometimes occur, especially in older people. Thus, proper joint surface restoration or stable fixation can be quite difficult to achieve using locking compression plates (LCPs). In such cases, joint replacement may be an option, always bearing in mind its high risk of complications, ranging from $11 \%$ to $45 \%$, especially in younger and active patients [1].

In cases of articular and multifragmented fracture associated with severe proximal ulna bone loss, massive bone allograft is another option to restore bone stock and to preserve joint function. Its use has been largely described with good results regard-

Received: April 6, $2021 \quad$ Revised: May 18, $2021 \quad$ Accepted: May 20, 2021

Correspondence to: Chiara Concina

Department of Orthopedics and Traumatology, Gorizia-Monfalcone Hospital, Via Galvani, 1 Monfalcone 34074, Italy

Tel: +39-04-8148-7255, E-mail: chiara.concina@asugi.sanita.f vg.it, ORCID: https://orcid.org/0000-0003-3891-840X

Financial support: None.

Conflict of interest: None.

Copyright@ 2021 Korean Shoulder and Elbow Society.

This is an Open Access article distributed under the terms of the Creative Commons Attribution Non-Commercial License (http://creativecommons.org/licenses/by-nc/4.0/) which permits unrestricted non-commercial use, distribution, and reproduction in any medium, provided the original work is properly cited. 
ing limb-sparing tumor resection, joint revision surgery, and infections [2]. Open elbow fracture-dislocations associated with severe bone loss are uncommon and rarely described. In this paper, we report the use of an ulna frozen massive allograft for an open Monteggia-like lesion with severe proximal ulna bone loss, associated with an ipsilateral ulnar shaft fracture, in an active patient.

\section{CASE REPORT}

This study was approved by the Institutional Review Board of Surgical Department ASUGI (IRB No. 0539-1256). The subject in this case signed an informed consent approving the discussion of his medical history in the present manuscript.

A 69-year-old man was admitted to our department after a high-speed motorcycle accident. The patient presented with large soft tissue damage in his right elbow with bone exposure (Fig. 1A); neither neurovascular deficits nor any other injuries were noted. He had no other comorbidities. He underwent elbow $\mathrm{X}$-rays that showed a Monteggia-like lesion with a multifragmented articular fracture of the proximal ulna with severe bone loss and an ipsilateral oblique fracture of the distal third of the ulnar shaft (Fig. 1B and C). This injury was classified as Gustilo-Anderson type IIIA.

We planned a two-stage strategy for the complex open injury. The patient was immediately prepared for the first surgery. Under general anesthesia, wound irrigation with saline and iodine solutions was performed, followed by surgical debridement and fracture-dislocation reduction and fixation using a bridging external fixator, according to damage control principles. Minimal fixation of the distal ulnar fracture fragments using an intramedullary K-wire and further stabilization of the radial head with a $\mathrm{K}$-wire were performed to achieve acceptable forearm alignment
(Fig. 2). The lateral ulnar collateral ligament (LUCL), radial collateral ligament (RCL), annular ligament, and medial collateral ligament (MCL) were completely torn and were repaired with simple sutures. The wound was closed without suture tension. Systemic antibiotic prophylaxis with amoxicillin/clavulanic acid and clindamycin was undertaken for 7 days, according to our hospital protocol with regards to open fractures.

Complete blood count formula, renal function, erythrocyte sedimentation rate (ESR), and C-reactive protein (CRP) were monitored weekly with a rapid decrease of the inflammation markers at 3 weeks post-surgery. Considering the high risk of infection in this complicated injury, and after a brief consultation with the infectious disease specialist, we continued oral amoxicillin/clavulanic acid ( $1.0 \mathrm{~g}$ four times a day) for a total of 12 weeks. Inflammation markers normalized in 8 weeks, and soft tissue
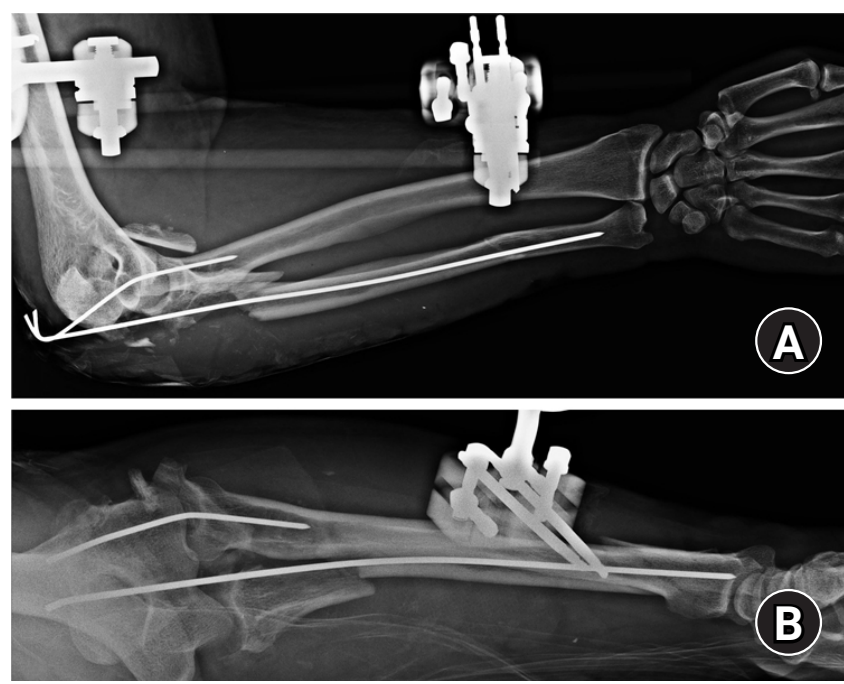

Fig. 2. (A, B) Radiographic control after damage control surgery with spanning external fixation and intramedullary K-wires.
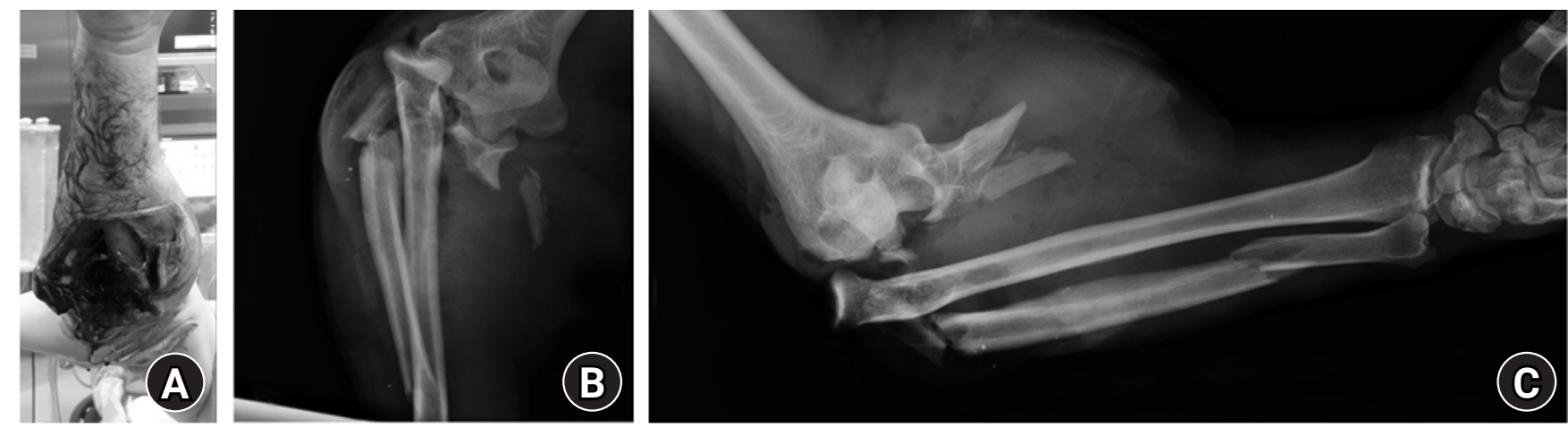

Fig. 1. (A) Open fracture and soft tissue damage classified as Gustilo-Andersen IIIA at patient arrival in the emergency department. (B, C) Preoperative radiographic control. 
condition improved such that a definitive surgical intervention could finally be planned at 3 months. During this time, the distal ulna fracture showed almost complete healing; however, nonunion of the residual proximal ulna was observed at 12 weeks follow-up. Given the comminuted fracture, the articular surface disruption, and the entity of the articular bone loss, we planned to use a massive ulna allograft fixed by plate and screws. We used an allogenic frozen proximal ulna from the Bone Bank per the international standard ISO 9001:2008 and under the European Guidelines of Tissue and Bone Banks.

The patient underwent the second surgery after 3 months under general anesthesia after having signed an informed consent. The external fixator was removed. We performed a posterior approach to the olecranon and the ulnar nerve was identified and preserved during the procedure. The triceps tendon was released from the residual fragments of the olecranon, exposing the proximal part of the forearm. We aspired to preserve the MCL, the LUCL, and their humeral attachments. The RCL, previously repaired during the first surgery, was tight; therefore, we did not perform any further procedure on the RCL. A synostosis between the proximal radius and the ulna was identified and thus removed via an ulna osteotomy was performed $8 \mathrm{~cm}$ from the tip of the olecranon, removing the proximal part of the ulna and the residual fragments. The ulnar allograft was prepared preserving the donor triceps tendon insertion, was implanted in the patient's elbow, and fixed with an eight-hole 2.7/3.5 LCP plate (DePuy Synthes, Warsaw, IN, USA). The novel ulnar-humeral joint was reduced and stabilized by two K-wires. The triceps tendon was then reattached on its insertion using a 5-mm titanium suture anchor (Healix, Mitek; DePuy, Warsaw, IN, USA) according to a modified Krackow suture technique and using the preserved donor triceps tendon to increase stability of the construct. The MCL, the LUCL, and the residual capsule were reattached to the graft using trans-osseous sutures. Good articular congruity was achieved. The wound was closed using a standard approach and a simple brace was used for 4 weeks. Postoperative standard radiographs of the elbow showed good positioning of the LCP plate with sufficient compression of the docking site and restoration of ulna-humeral joint congruity (Fig. 3).

The two K-wires were removed 5 weeks after surgery to start rehabilitation. Ten days after removal, a posterior wound dehiscence with siero-hematic effusion appeared. Although no infectious agents were identified, levofloxacin $500 \mathrm{mg}$ twice a day and rifampicin $600 \mathrm{mg}$ were administered for 12 weeks, after a brief consultation with the infectious disease specialist. In addition, superficial wound debridement was conducted. V.A.C. (Vacuum Assisted Closure, Acelity; KCI, St. Paul, MN, USA) negative pres- sure wound therapy was applied for 3 weeks, as well as PICO (Smith \& Nephew, Watford, UK) negative pressure wound therapy for an additional 7-week period, achieving complete skin closure. CRP and ESR levels were found to be in range at 8 weeks and the antibiotic therapy was well-tolerated by the patient during the entire treatment period.

The patient was assessed 3 years after the second surgery. At examination, the soft tissues looked normal and the range of motion of his elbow was $110^{\circ}$ of flexion, $30^{\circ}$ of extension, $10^{\circ}$ of supination, and $0^{\circ}$ of pronation and the patient had already returned to his normal daily activities with limitation concerning weightlifting of heavy objects and some residual pain after work activities (Fig. 4). No pain was present at rest or during any flexion/extension movement related to simple daily activities. The Disabilities of the Arm, Shoulder and Hand (DASH) score was 16.7. There was no medial instability. Mild discomfort and apprehension during the lateral pivot-shift test and posterolateral rotatory drawer test suggested mild posterolateral rotatory instability. Radiographs at 3 years showed good allograft osteointegration without any signs of bone reabsorption on the docking point or hardware loosening or severe osteoarthritis of the radial-humeral joint with partial lateral humeral condyle reabsorption (Fig. 5A and B). Computed tomography (CT) scans confirmed the radiographic findings and good creeping substitution at the docking site (Fig. 5C).

\section{DISCUSSION}

Elbow fracture-dislocations can lead to osteoarthritic changes, articular stiffness, and recurrent instability. These injuries are typically addressed with compressive and locking plates, regardless of whether associated with capsular-ligament repair or reconstruction. When the fracture is comminuted, and the articular surface is severely impaired, joint restoration becomes diffi-
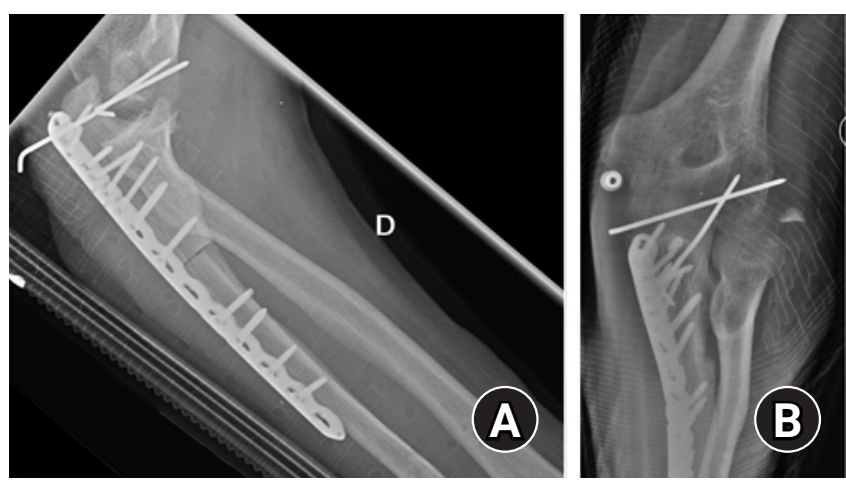

Fig. 3. (A, B) Radiographic control after definitive surgery and open reduction and internal fixation with the massive ulna allograft. 

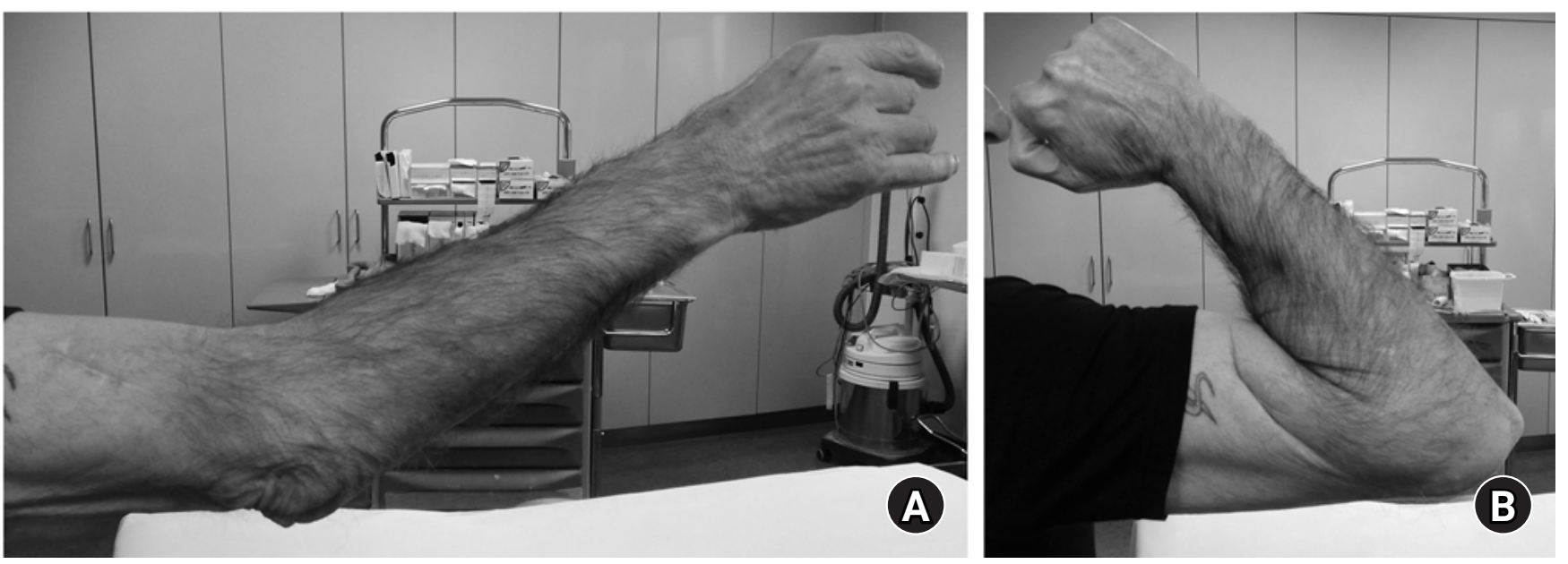

Fig. 4. (A, B) Clinical assessment of the patient at 3 years after surgery with satisfactory clinical outcome.
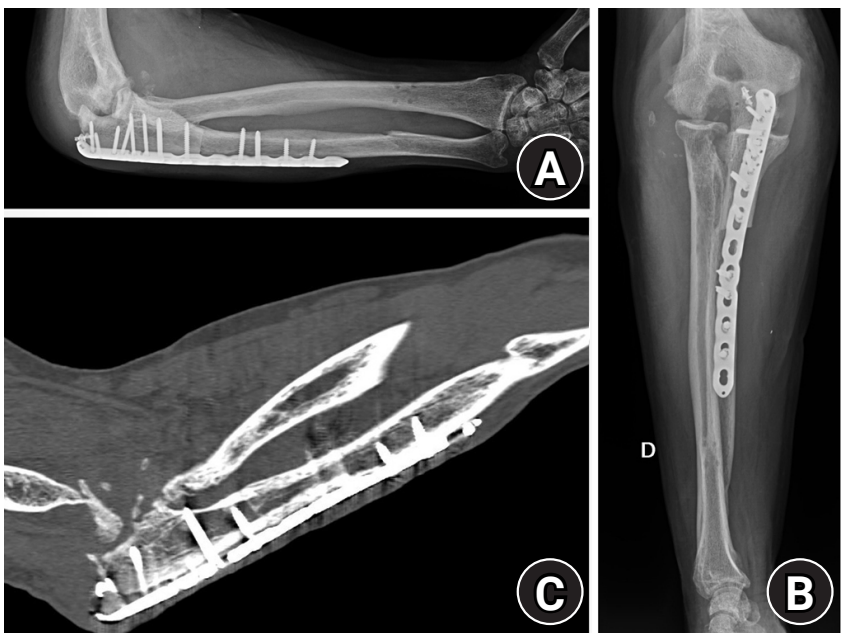

Fig. 5. (A, B) Radiographic control at 3 years shows good articular congruence, healing of the docking point, and advanced arthritic degeneration on the humeral-radial site. (C) Computed tomography scan at 3 years confirms healing between the ulna and allograft at the docking site by creeping substitution.

cult to achieve and soft tissue biology is often compromised. In such cases, open reduction and internal fixation is not always possible, and is often associated with high risk of hardware failure and patient functional insufficiency.

As in other articular injuries, joint replacement can be used. Several authors reported good clinical and radiographic results in total elbow arthroplasty in $\mathrm{AO}$ type $\mathrm{C}$ distal humeral fractures in elderly patients with osteoporotic bone [3]. Barco et al. [4] reported a survivor rate of $85 \%$ at 5 years and $76 \%$ at 10 years follow-up in rheumatoid geriatric patients treated with primary total elbow replacement for distal humeral fractures. However, when this survivor rate is compared to hip and knee replacement, survivor rate is undoubtedly lower; in fact, the complication rate for total elbow replacement is higher in comparison to other joint arthroplasties. Complications are also more frequent in young, obese, and smoking patients, and that functional recovery is better in rheumatic elbows than in the fractured ones [5].

In our 69-year-old patient, an elbow mega prosthesis should have been used due to the large ulnar bone defect. Even though Capanna et al. [6] described good results in 31 oncological patients when such technique was used, an elbow mega prosthesis was not a suitable option for our patient. In this case, the patient would have a high risk of implant loosening related to the poor ulnar bone stock and to his high functional request, considering that the injured arm was his dominant one, and he would have been at high risk of infection due to the previous severe soft tissue damage. Therefore, we decided to use a massive osteochondral allograft considering the proximal ulna bone loss and the need to provide the best functional restoration, as is typically required for active patients. Even if the elbow is an infrequent site for tumor and metastasis, the use of massive bone allograft for limb-sparing tumor resection is widely described in the literature [7], especially for hip and knee surgery. The main advantage for a massive allograft is the possibility to totally restore the bone stock while maintaining joint function. In our opinion, this approach should safely be considered in active patients.

Preoperative planning is fundamental. In fact, a mismatch between the articular portion of the allograft and the host trochlea can bring elbow instability and cartilage wear, because of the altered mechanical stress on the joint surface. Preoperative CT scans and contralateral elbow radiographs helped in measuring the trochlea size and finding a suitable ulnar allograft. Allograft storage protocol is also important to ensure good cell biology and 
matrix content. Freezing and sterilization reduce the cellular component [8]. In our case, the graft had been frozen and preserved at $-80^{\circ} \mathrm{C}$ prior to surgery, according to the Bank of Tissues protocols.

Massive bone allograft is not complication free. Fractures, infections, non-unions, articular degeneration, and joint instability are frequently described in the literature, reporting an overall complication rate that ranges from $40 \%$ to $70 \%$ [2]. Allograft fractures were seen in $10 \%$ to $52 \%$ of the cases [2] and usually occur after graft healing and with little to no trauma. These fractures are probably associated with incomplete creeping substitution, and the larger the osteochondral allograft, the higher the risk of fracture can be.

In our patient, neither allograft fractures nor graft-host nonunions occurred. We should consider, however, that massive bone allograft is generally used alone or in an allograft-prosthesis composite for oncological patients who underwent perioperative chemotherapy or radiotherapy. This can also explain their high complication rate. In our case, we operated on a non-oncological patient. In addition, the ulna is not a weight-bearing bone, so the mechanical stress on this bone is much lower.

Graft fracture can also depend on fixation technique. In our patient, we used an LCP plate that allows a rigid and stable fixation and reduces the risk of fracture and allows compression and contact at the docking site. These considerations are crucial to ensure healing of the graft-host junction, while avoiding nonunions.

Infection is the most feared problem for orthopedic surgeons; in fact, it is the most common complication associated with graft removal in the first 2 or 3 years after reconstruction [9]. Infections are reported in up to $16 \%$ of previous case cohorts [2,9]. There is no consensus about the management of massive allograft infections. A topic of debate is whether to remove the graft, considering that graft removal might lead to severe dysfunction, and in some cases limb amputation. Aponte-Tinao et al. [10] retrospectively analyzed 673 patients who underwent reconstruction with massive bone allografts for tumors or for a previous limb rescue procedure. Only $18 \%$ of the infected patients were treated successfully with surgical debridement and antibiotics without graft removal; the remaining $82 \%$ of patients were treated with graft removal, a cemented spacer, and a second reconstruction and $34 \%$ of the subjects presented with new infections. In our patient, the infection was superficial in a way that wound debridement, negative pressure therapy, and antibiotics for 3 months were enough to achieve wound healing. The use of negative pressure therapy is well documented in the literature, although the mechanism remains unclear. It has been suggested that it promotes wound healing by fluid absorption when in excess, by preserving microcirculation dynamics through toxin removal from the surrounding tissue, and by decreasing the bacterial load in the case of infection.

Time lapse between trauma and bone allograft is another point to consider. Given the few case reports of allograft for knee fractures described in literature, at the moment there are no guidelines that define the proper time of action. In our case report, 3 months were necessary before definitive surgery. In fact, even if there were not any signs of infection, ESR and CRP normalized only 8 weeks after surgery. Because timing is crucial to reduce the risk of infection, we suggest that bone allograft after complete wound healing and normalized laboratory exams are mandatory. Moreover, a closer collaboration between the orthopedic surgeon and the infectious disease specialist is fundamental for deciding timing for the second stage after the first surgical treatment, as well as the duration of antibiotic therapy after allograft implantation. In some cases, when the soft tissues are significantly injured, and muscle flaps are required to cover the massive allograft, a plastic surgeon can be very helpful when available.

Another key point of these fracture-dislocations is the management of capsular-ligament injuries that are usually severe, as in our case. Ligament repair or reconstruction should be done to avoid instability. In fact, even if the osseous anatomy of the ulnohumeral joint gives intrinsic stability acting as a hinged joint, MCL and LCL complex are primary static constraints.

Open elbow fracture-dislocations are particularly challenging injuries. Massive ulnar osteochondral allograft can be considered a valid option in cases of open Monteggia-like lesions with large bone defects. Based on our study, this procedure restores complete joint function and offers a satisfactory bone stock for a future total elbow replacement.

\section{ORCID}

Chiara Concina https://orcid.org/0000-0003-3891-840X Marina Crucil https://orcid.org/0000-0001-6673-4435

Emmanouil Theodorakis https://orcid.org/0000-0002-0321-4608 Giorgio Saggin https://orcid.org/0000-0003-1660-5461

Silvia Perin https://orcid.org/0000-0002-0862-0795

Franco Gherlinzoni https://orcid.org/0000-0003-4759-7257

\section{REFERENCES}

1. Voloshin I, Schippert DW, Kakar S, Kaye EK, Morrey BF. Complications of total elbow replacement: a systematic review. J Shoulder Elbow Surg 2011;20:158-68. 
2. Matejovsky Z Jr, Matejovsky Z, Kofranek I. Massive allografts in tumour surgery. Int Orthop 2006;30:478-83.

3. Schindelar LE, Rondon AJ, Ilyas AM. Total elbow arthroplasty versus open reduction and internal fixation for the management of distal humerus fractures in the elderly. Orthopedics 2019;42:22-7.

4. Barco R, Streubel PN, Morrey BF, Sanchez-Sotelo J. Total elbow arthroplasty for distal humeral fractures: a ten-year-minimum follow-up study. J Bone Joint Surg Am 2017;99:1524-31.

5. Kwak JM, Koh KH, Jeon IH. Total elbow arthroplasty: clinical outcomes, complications, and revision surgery. Clin Orthop Surg 2019;11:369-79.

6. Capanna R, Muratori F, Campo FR, et al. Modular megaprosthesis reconstruction for oncological and non-oncological resection of the elbow joint. Injury 2016;47 Suppl 4:S78-83.
7. Muscolo DL, Ayerza MA, Aponte-Tinao LA. Massive allograft use in orthopedic oncology. Orthop Clin North Am 2006;37: 65-74.

8. de Girolamo L, Ragni E, Cucchiarini M, van Bergen CJ, Hunziker EB, Chubinskaya S. Cells, soluble factors and matrix harmonically play the concert of allograft integration. Knee Surg Sports Traumatol Arthrosc 2019;27:1717-25.

9. Aponte-Tinao LA, Ayerza MA, Albergo JI, Farfalli GL. Do massive allograft reconstructions for tumors of the femur and tibia survive 10 or more years after implantation? Clin Orthop Relat Res 2020;478:517-24.

10. Aponte-Tinao LA, Ayerza MA, Muscolo DL, Farfalli GL. What are the risk factors and management options for infection after reconstruction with massive bone allografts? Clin Orthop Relat Res 2016;474:669-73. 